\title{
Myocardial Area at Risk and Salvage in reperfused acute MI Measured by Texture Analysis of Cardiac T2 Mapping and Its Prediction Value of Functional Recovery in the Convalescent Stage
}

\author{
Zi-yang Fan \\ Shanghai Jiao Tong University School of Medicine Affiliated Renji Hospital \\ Chong-wen Wu \\ Shanghai Jiao Tong University School of Medicine Affiliated Renji Hospital \\ Dong-Aolei An \\ Shanghai Jiao Tong University School of Medicine Affiliated Renji Hospital \\ Bing-hua Chen \\ Shanghai Jiao Tong University School of Medicine Affiliated Renji Hospital \\ Luke Wesemann \\ Wayne State University School of Medicine \\ Ting Yue \\ Shanghai Jiao Tong University School of Medicine Affiliated Renji Hospital \\ Jie He \\ Shanghai Jiao Tong University School of Medicine Affiliated Renji Hospital \\ Jia-ni Hu \\ Wayne State University School of Medicine \\ Jun Bu \\ Shanghai Jiao Tong University School of Medicine Affiliated Renji Hospital \\ Jian-rong Xu \\ Shanghai Jiao Tong University School of Medicine Affiliated Renji Hospital \\ Lian-Ming Wu ( $\nabla$ wimssmu@126.com) \\ Shanghai Jiao Tong University School of Medicine Affiliated Renji Hospital Department of Laboratory Medicine
}

\section{Research Article}

Keywords: Texture Analysis, T2 Mapping, Area at Risk

Posted Date: February 12th, 2021

DOl: https://doi.org/10.21203/rs.3.rs-218147/v1

License: @ (i) This work is licensed under a Creative Commons Attribution 4.0 International License. Read Full License

Version of Record: A version of this preprint was published at The International Journal of Cardiovascular Imaging on July 19th, 2021. See the published version at https://doi.org/10.1007/s10554-021-02336-7. 


\section{Abstract}

To assess the ability of distinguishing the area at risk (AAR) and evaluating the prognostic abilities of T2-mapping texture analysis (TA) in reperfused acute $\mathrm{MI}, 106$ patients who were diagnosed with $\mathrm{AMI}$ and treated with percutaneous coronary intervention (PCl) underwent acute (less than five days) enhanced cardiac magnetic resonance imaging. Of these patients, 45 of them had a subsequent CMR scan following recovery (after at least three months). Cine imaging, T2-Mapping, T2-weighted STIR imaging, and LGE imaging were performed. In the TA, regions of interest (infarcted, salvageable, and remote) were drawn by two blinded, independent readers based on LGE and T2-weighted imaging. Seven independent texture features on T2-Mapping were selected: Perc.50\%, S(2,2)InvDfMom, S(2.-2)AngScMom, S(4,0)Entropy, 45dgrLngREmph, 45dgr_Fraction and 135dr_GLevNonU. Among them, 45dgr_LngREmph, 45dgr_Fraction and 135dr_GLevNonU showed more promise. The average value of 135dr_GLevNonU in the infarct zone, AAR zone, and the remote zone was: $61.96 \pm 26.03,31.811 \pm 18.933$ and $99.839 \pm 26.231$, respectively. Additionally, $135 \mathrm{dr}$ GLevNonU provided the highest $0.855( \pm 0.083$ ) area under the curve (AUC) from the receiver operating characteristic curve (ROC curve) for distinguishing AAR from the infarct zone. The AUC for differentiating AAR from the remote zone is $0.942 \pm 0.041$. Texture features are not associated with convalescent decreased strain or ejection fraction $(E F)(p>0.05)$ in the standard regression analysis; and cannot predict left ventricle remodeling (LVR) in the logistic regression analysis ( $p>0.05)$. T2-mapping TA in reperfused AMI can distinguish AAR from both the infarct zone and the remote myocardial zone without LGE imaging. However, these features cannot predict patients' functional recovery in the convalescent stage.

\section{Introduction}

Myocardial infarction (MI) is necrosis of the muscular layer of the heart caused by an imbalance between oxygen supply and myocardial demand. Any condition that suddenly blocks blood flow to the heart is referred to as an acute coronary syndrome (ACS). ACS is estimated to occur in more than 370,000 deaths a year in the U.S. [1]. Cardiac MR (CMR) has many advantages to offer patients who may have a MI, such as those with ACS. CMR can provide functional assessment, cardiovascular anatomy, and characterization of myocardial tissue with high reproducibility and accuracy. Late gadolinium enhancement (LGE) imaging is the current gold standard for the assessment of ischemic myocardial scars, and T2-STIR can aid in the detection of myocardial edema.

The prevalence of patients with coronary artery disease would be higher if they also suffered from any of the chronic kidney diseases[2]. However, some patients with CKD cannot undergo gadolinium administration, which makes LGE imaging unavailable. Additionally, the measurement of AAR and final infarct size (FIS) is vital for the complete evaluation of MI, giving a better idea of how much ischemic myocardium can be salvaged[3]. FIS and AAR are obtained from T2-STIR and LGE imaging.

T2-STIR plays an essential role in defining the AAR prior to troponin elevation. However, the problem of T2-STIR, such as unreliability and image registration that is only functional with LGE imaging, has limited its widespread use[4]. T2 mapping overcomes these limits. T2 mapping is a nonenhanced sequence; thus, it's available even to CKD patients. It can be used as a technique to reflect the calculated T2 relaxation time, which can be analyzed quantitatively, whereas T2-STIR cannot. T2 mapping gives access to nominally measured values for T2. Compared to T2 values for normal myocardial tissue, the T2 relaxation time of infarct zone and AAR will be longer. Evidence shows that the relaxation time of the infarct zone is longer than AAR, indicating the possibility of distinguishing between salvaged and infarcted myocardium[5].

The T2 mapping sequence contains more information than what has been aforementioned. Texture analysis (TA) is a branch of image science, from which we can obtain a wide range of underlying texture data. By analyzing these data, TA has potential diagnostic and prognostic value[6], and its ability to provide massive texture features has drawn much attention in the field of medical imaging. It has been recently reported in the literature as having the capacity of texture analysis in the domains of oncologic, neuroradiologic, musculoskeletal, and lung imaging[2]. Concerning cardiovascular imaging, TA on nonenhanced T1-weighted images allows for the assessment of the variations seen in myocardial MR imaging of hypertrophic cardiomyopathy (HCM) with excellent accuracy[7].

Thus, our study is aimed at the detection of acute infarcted myocardium, distinguishing AAR and FIS with nonenhanced T2 mapping texture analysis. Furthermore, we hypothesize that T2 mapping using TA can predict the prognosis of AMI patients and further elucidate the relationship between TA features and cardiac functional features.

\section{Methods}

\section{Study population}

This study included 106 patients (87 males, mean age $61 \pm 11$ years) with AMI diagnosed according to guidelines and treated with PPCl (Table.1). Fig.1 showed patient recruitment, inclusion and exclusion criteria for the study. Patients underwent CMR imaging at 3.0 Tesla within 1 to 7 days after AMI. 45 of these patients underwent a follow-up CMR scan on the same MR machine after at least three months (mean time 102d). Exclusion criteria for patients were: i) history of myocardial infarct, ii) lack of necessary CMR sequences, iii) interruption of T2-STIR, T2 mapping, or LGE imaging by artifacts.

\section{CMR imaging data acquisition}

The CMR scan was performed on a 3.0T system. (Ingenia, Philips Healthcare, Best, The Netherlands). Cine sequences were performed by balanced SteadyState Free Pression, bssFP, breath-hold technique, and with retrospective ECG-triggering in short axis (SAX). 10 to 12 slices of SAX, 2ch, 3ch, and 4ch LAX were performed. T2-STIR and LGE imaging of SAX slices were correlated to cine imaging to ensure they were in the same position. LGE images were scanned 
10 15 minutes after the contrast medium was injected. The 3 SAX slices of T2 mapping were basal, mid, and apical. The contrast medium was Magnevist (Bayer, German), the dose was $0.15 \mathrm{mmol} / \mathrm{kg}$, injection speed was $3.0 \mathrm{ml} / \mathrm{s}$.

\section{CMR imaging data analysis and subgroups}

CMR studies were analyzed offline by an experienced radiologist using CVI42 (Circle Cardiovascular Imaging Inc., Calgary, Alberta, Canada). LVEF (\%), LVEDV $(\mathrm{ml})$, LVESV $(\mathrm{ml})$ and LV mass $(\mathrm{g})$ were assessed by cine imaging. The area of edema was identified as "T2WI+2SD" and the infarcted area was identified in LGE imaging with full-width at half-maximum (FWHM) technique[8]. In order to ensure the accuracy of the image position correlation within LGE, T2-STIR, and T2 mapping, the slice of SAX was determined by T2 mapping. ROls on T2 mapping were drawn by hand according to T2-STIR and LGE (Fig.2). Three ROIs were defined as follows: i) infarcted: positive on LGE; ii) salvageable: positive on T2-STIR but negative on LGE; iii) remote: negative on both T2-STIR and LGE.

The patients were divided into two groups, with (40) or without (66) microvascular obstruction (MVO), according to LGE imaging, to prevent the potential influence on T2 mapping texture analysis.

\section{Texture analysis selection and dimension reduction}

ROIs were drawn by two independent researchers; texture features were extracted by a freely available software (MaZda version 4.6, Institute of Electronics, Technical University of Lodz, Lodz, Poland). An intraclass correlation coefficiency (ICC) test was done to these two researchers' data to assess the stability of the features[9]. All features (Online Appendix Table 1)extracted from MaZda went through the ICC test and were considered stable ( $r \geq 0.75$ ). The selected stable features were then filtered by Boruta, which is a "random forest" algorithm. It randomly generates features and calculates their correlation with ROI, and then a top-down search was done to find all relevant features by comparing the results of previous calculations with our T2 mapping extracted features.

Considering the collinearity between these features, we divided features into separate groups. In each group, the most significant features in Boruta were selected for the final analysis. If T2 mapping means were eliminated during the filter, we would still add it to the final analysis. These procedures were performed in R 3.6.0 with RStudio.

\section{Statistical analysis}

For the selected features, the diagnostic accuracy of distinguishing AAR from the infarct zone was evaluated from ACU from the ROC curve in both subgroups to assess whether MVO affects the differentiation.

Each correlation of the TA features towards decreased EF and strain were assessed by univariable linear regression. For follow up scan patients, the following variables were considered positive for left ventricular remodeling: i) a $10 \%$ decrease of EF or, ii) a $20 \%$ increase of EDV and a $5 \%$ decrease of EF. Logistic regression analysis was then performed.

In this step, statistical analysess were carried out using SPSS 20.0 (SPSS Inc., Chicago, USA).

Our study protocol was approved by the Ethics Committee of ** Hospital.

\section{Results}

\section{Texture feature selection}

After the ICC test, 58 of 300 features were considered stable and entered the Boruta algorithm. The results from Boruta are presented in Fig.3. 49 features were considered more significant towards ROI compared to randomly generated features, which then entered further analysis.

To address the limitation of the Boruta algorithm not detecting collinearity between features, we performed a correlation matrix that included the 49 mostsignificant features to find allhighly collinear ones (Fig.4) [7]. From the highly correlated parameters (defined as Pearson's $r \geq 0.6$ ), only one from each cluster (7 in total) showed the highest impotency towards ROI differentiation during the Boruta test and was used for further analysis.

Though the T2-mapping mean was eliminated during measurement, we did use the mean for further analysis. The eight important and independent features were Mean, Perc.50\%, S(2,2)InvDfMom, S(2.-2)AngScMom, S(4,0)Entropy, 45dgrLngREmph, 45dgr_Fraction and 135dr_GLevNonU (Fig.5). Each containing three co-occurrence matrices and three run-length matrices.

\section{Selected feature statistics}

In each group, texture features were presented with mean \pm SD (Table 2). Between the two subgroups, all eight features showed no differences ( $p>0.05$ ). The value distribution can be seen in Fig.6. Other than mean and Perc.50\%, the distribution of values was similar with or without MVO.

The average mean values of T2 mapping in all patients (106) in the infarct zone, AAR, and remote zone were $71.19 \pm 15.93,64.191 \pm 14.699$, and $56.517 \pm 10.379$, respectively. The $\mathrm{T} 2$ relaxation times of all three areas were longer than the corresponding $\mathrm{T} 2$ relaxation times in the healthy myocardium in previous reports[10]. A progressive increase is evident in the T2 mapping mean. However, in the subgroup of patients with MVO, the value between the infarct zone $(67.627 \pm 17.048)$ and AAR $(66.105 \pm 12.215)$ would be rather indistinguishable $(p>0.001)$. This may be because MVO causes a decrease in T2 relaxation time, but a lengthening of time during edemaThus, distinguishing the infarct zone from AAR is infeasible using only T2 relaxation time. The feature $135 \mathrm{dr}$ _GLevNonU showed differences between each subgroup $(p<0.001)$. 
Among the features, $135 \mathrm{dr} \_G L e v N o n U$ was remarkable. The average value in the infarct zone, AAR, and remote zone were: $61.96 \pm 26.03,31.811 \pm 18.933$ and $99.839 \pm 26.231$, which provided a $0.845( \pm 0.052)$ AUC from ROC for distinguishing AAR from the infarct zone in all 106 patients (Table 3 , Online Appendix Fig.1). This feature was remarkably stable in both subgroups ( $0.855 \pm 0.083$ in patients with MVO and $0.845 \pm 0.066$ in patients without MVO), and could distinguish the remote area and AAR more accurately in all groups (AUC $0.980 \pm 0.015$ ). By dividing the affected myocardium in the T2 mapping image and extracting 135dr_GLevNonU, the infarcted zone, AAR, and the remote zone would all be separated.

As mentioned above, 135dr_GLevNonU is a run-length matrix, describing Grey level non-uniformity. High grey-level non-uniformity values represent a nonuniform texture, i.e., heterogeneity[11]. This means that compared to the remote and infarcted zone in the myocardium, grey-level in AAR showed more homogeneity.

Other potential features were 45dgr_LngREmph and 45dgr_Fraction, which are both also run-length matrices. A higher value of long-run emphasis means more homogeneity of the gray-level image, while a higher value of Fraction means a more significant variation of the gray-level image[12]. These two features would also separate AAR from the remote zone. (AUC $0.957 \pm 0.023$ and $0.934 \pm 0.034$ ).

\section{Texture features in predicting prognosis}

To analyze whether the selected features could determine the prognosis of AMI patients, we measured cardiac function index for 45 follow-up patients. The baseline average EF was $48.42 \% \pm 11.7 \%$, and the follow-up EF was $49.51 \% \pm 12.0 \%$. The average baseline global circumferential strain was $11.84 \%$.

First, we performed a univariable linear regression to examine the correlation between decreased EF and decreased global circumferential strain. The decreased strain was corrected by the baseline data: the difference between the follow-up and baseline strain were divided by the baseline data. Univariable linear regression indicated none of the features could predict the strain or EF in the follow-up CMR scan. Furthermore, we investigated the correlation between mid-circumferential strain, radial strain, and longitudinal strain. Unfortunately, the linear regression correlation with texture features was very poor ( $p>0.05$ ).

In our left ventricular remodeling assessment, 14 of 45 (31.11\%) patients were identified as positive. Logistic regression analysis showed none of the selected texture features were significantly associated with left ventricular remodeling (pष0.05).

\section{Discussion}

In this study, we found that T2 mapping texture analysis could both differ and quantify AAR in AMI patients. Selected texture features were confirmed to associate with AMI myocardium ROI during the Boruta step, and the most significant features went through to further analysis. The values of texture features in all three major zones were measured. The features could differ between the infarcted zone, AAR, and the remote zone, showing that T2 mapping texture analysis could discover AAR without enhancement. However, the correlation between MI prognosis and selected features were not found.

It can be challenging for AMI patients to undergo comprehensive CMR imaging because of the demand for multiple breath-holds. Furthermore, enhanced CMR is not available for CKD or any patient with high serum creatinine. Morbidity and mortality risk will increase in patients with end-stage renal failure (ESRF) who also suffer from cardiovascular diseases. Nearly half of all deaths in hemodialysis (HD) patients were associated with cardiovascular lesions[13, 14]. Therefore, nonenhanced cardiac T2 mapping sequences, which may improve clinical practicability, meet this need.

A recent study demonstrated T1 cut-off values for edematous versus necrotic myocardium were identified as $1251 \mathrm{~ms}$ and $1400 \mathrm{~ms}$ on a $3 \mathrm{~T}$ MR system [15]. Additionally, another study claimed that native T1 mapping is a remarkably adapted technique in HD patients. With HD patients' common changes in fluid status, native T1 values seem to be stable[16]. According to an inter-center study, native T1 mean (ms) has a bias between medical centers, and T1 mapping may have difficulties in isolating MVO. From our study, T2 mapping texture analysis is reliable for HD patients. As mentioned above, the AUC for 135dr_GLevNonU distinguishing AAR from the remote zone is $0.980 \pm 0.015$, which was very promising and more stable than native T1.

Another optional method to assess HD patients' cardiac tissue characterization is deep learning. In a recent study focused on diagnosing chronic MI on nonenhanced cine CMR using deep learning, an AUC of 0.89 was achieved by Nan Zhang et al [17]. Another texture analysis study indicated nonenhanced cine MR images can diagnose subacute and chronic MI with high accuracy (AUC of 0.93 and 0.92) [14]. Our study achieved an AUC of 0.98 between AAR and the remote myocardial zone in AMI patients. Differentiating AAR from the remote zone reflected its ability to diagnose acute myocardial infarction. As part of nonenhanced CMR sequences, T2 mapping provided more information than cine MR.

A recent animal study validated that T2-STIR and contrast-enhanced steady-state free precession MRI (CE-SSFP) allow for unbiased quantification of AAR in the presence of MVO versus histopathology in the subacute phase of MI [18]. Demonstrating that T2-STIR and CE-SSFP were valuable in quantifying AAR. This method may reduce CMR duration and benefit patients with poor breath-holding ability.

Our study is not the first to attempt to estimate the prognostic potential of texture analysis. Recently, a study investigating the ability of acute infarct ECV and the acute transmural extent of LGE to predict convalescent function demonstrated that ECV had higher accuracy than LGE in predicting improved wall motion (AUC was 0.77 versus $0.66 ; p=0.02$ ) [19]. In the same study, univariable regression analysis predicted that infarct ECV was significantly associated with convalescent strain and convalescent $E F(p<0.0005$ and $P=0.001)$.

Our study failed to find a correlation between features and LV function. Among the 300 features extracted by MaZda, one specific feature relevant to both AAR and prognosis was not found. This might suggest that in further research, it is important to select variables that contribute to decreased EF or strain from the Boruta algorithm filter. 
Therefore, texture analysis in CMR (some sequences we believe) could predict functional recovery following AMI. Findings of T1 mapping support the prediction of LV function using radiomics.

Radiomics is promising in other heart diseases such as dilated cardiomyopathy (DCM), myocarditis, and hypertrophic cardiomyopathy (HCM). A recent study showed that most histogram features in T1 mapping TA were higher in the DCM group when compared to healthy controls. Their diagnostic accuracy when using the support vector machine classifier with all these histogram and gray-level co-occurrence matrix features was $0.85 \pm 0.07$.[20]

In another texture analysis MI study, researchers found that patients would have higher kurtosis (coarse-scale, $p=0.005)$ and lower skewness (fine-scale, $p=$ 0.046) if they suffered from arrhythmic events during the follow-up period [21]. Reflecting LGE imaging, textural heterogeneity was significantly associated with arrhythmic events.

Numerous choices of CMR sequences and features among each sequence indicates that radiomics in CMR is still a rather promising field and requires further exploration. Radiomics may have the potential to benefit many patients by making diagnoses and prognoses more accurate. Hopefully, radiomics in CMR will become a diagnostic gold standard in various cardiac diseases.

We must acknowledge several limitations of our study. Our study used retrospective data from a single vendor and a single center. This study focused on T2 mapping TA, thus to extract data, researchers must draw ROIs on one affected SAX slice. Differentiating AAR was reliable using only one image slice, but when it comes to predicting the complete heart function index, one-slice-data might be inadequate. On another aspect, in our CMR scan, T2 mapping was performed using three slices SAX, myocardium information of basal, mid, and apex was gathered. According to recent research, to accurately quantify the AAR by T1 and T2 mapping, full left ventricular coverage is essential[22]. Conditions permitting, further research focused on AAR should take full left ventricular coverage. Furthermore, all the strain data in our study was whole-hearted, or from mid slice circumferential. These represented whole heart myocardial prognosis but were not accurately correlated to the selected T2 mapping slice or the American Heart Association (AHA) segments that AMI affected. This may cause the independence between texture features and strain. Maybe there's a hidden TA feature associated with the affected AHA segment strain but not correlated with AAR, which requires further investigation.

This study focused on the application of T2 mapping on acute and convalescent CMR scans on MI patients. Our analysis provided evidence arguing that T2 mapping is a favorable method quantifying AAR. Nonenhanced MR imaging and shortened scan times largely widened T2 mapping's versatility. Assessment of radiomics studies have continuously proven to be valuable in heart diseases. Whether T2 mapping could predict LV function in MI patients needs further investigation.

\section{Declarations}

\section{Acknowledgement}

We acknowledge the assistance from Rui Wu, Ruo-yang Shi and Yi-si Dai, who provided a lot of help in data processing.

\section{Grant Support}

Supported by National Natural Science Foundation of China (No.81873886 and No.81873887) \ Shanghai Shenkang Hospital Development Center Clinical Research and Cultivation Project $₫$ SHDC12018X21『; Shanghai Science and technology innovation action plan, technology standard project (19DZ2203800); Shanghai Jiao Tong University school of medicine Double hundred outstanding person projrect (20191904); Shanghai Jiao Tong University medical cross project YG2017QN44.

\section{References}

[1]. Moore, A., et al., Acute Myocardial Infarct. Radiol Clin North Am, 2019. 57(1): p. 45-55.

[2]. Baessler, B., et al., Subacute and Chronic Left Ventricular Myocardial Scar: Accuracy of Texture Analysis on Nonenhanced Cine MR Images. Radiology, 2018. 286(1): p. 103-112.

[3]. Kim, H.W., et al., Relationship of T2-Weighted MRI Myocardial Hyperintensity and the Ischemic Area-At-Risk. Circ Res, 2015. 117(3): p. 254-65.

[4]. Lota, A.S., P.D. Gatehouse and R.H. Mohiaddin, T2 mapping and T2* imaging in heart failure. Heart Fail Rev, 2017. $22(4):$ p. $431-440$.

[5]. Hammer-Hansen, S., et al., Distinction of salvaged and infarcted myocardium within the ischaemic area-at-risk with T2 mapping. Eur Heart J Cardiovasc Imaging, 2014. 15(9): p. 1048-53.

[6]. Baessler, B., et al., Cardiac MRI and Texture Analysis of Myocardial T1 and T2 Maps in Myocarditis with Acute versus Chronic Symptoms of Heart Failure. Radiology, 2019. 292(3): p. 608-617.

[7]. Baessler, B., et al., Texture analysis and machine learning of non-contrast T1-weighted MR images in patients with hypertrophic cardiomyopathyPreliminary results. Eur J Radiol, 2018. 102: p. 61-67.

[8]. Khan, J.N., et al., Comparison of semi-automated methods to quantify infarct size and area at risk by cardiovascular magnetic resonance imaging at $1.5 \mathrm{~T}$ and 3.0T field strengths. BMC Res Notes, 2015. 8: p. 52. 
[9]. Khan, J.N., et al., Comparison of cardiovascular magnetic resonance feature tracking and tagging for the assessment of left ventricular systolic strain in acute myocardial infarction. Eur J Radiol, 2015. 84(5): p. 840-8.

[10]. Wang, J., et al., Native T1 and T2 mapping by cardiovascular magnetic resonance imaging in pressure overloaded left and right heart diseases. J Thorac Dis, 2018. 10(5): p. 2968-2975.

[11]. Kim, H.S., et al., Preoperative CT texture features predict prognosis after curative resection in pancreatic cancer. Sci Rep, 2019. 9(1): p. 17389.

[12]. Fang, W.H., et al., Resectable pancreatic ductal adenocarcinoma: association between preoperative CT texture features and metastatic nodal involvement. Cancer Imaging, 2020. 20(1): p. 17.

[13]. de Jager, D.J., et al., Cardiovascular and noncardiovascular mortality among patients starting dialysis. JAMA, 2009. 302(16): p. 1782-9.

[14]. Baessler, B., et al., Subacute and Chronic Left Ventricular Myocardial Scar: Accuracy of Texture Analysis on Nonenhanced Cine MR Images. Radiology, 2018. 286(1): p. 103-112.

[15]. Garg, P., et al., Role of cardiac T1 mapping and extracellular volume in the assessment of myocardial infarction. Anatol J Cardiol, 2018. 19 (6): p. 404-411.

[16]. Graham-Brown, M.P., et al., Native T1 mapping: inter-study, inter-observer and inter-center reproducibility in hemodialysis patients. J Cardiovasc Magn Reson, 2017. 19(1): p. 21.

[17]. Zhang, N., et al., Deep Learning for Diagnosis of Chronic Myocardial Infarction on Nonenhanced Cardiac Cine MRI. Radiology, 2019. 291 (3): p. 606-617.

[18]. Hansen, E., et al., Validation of contrast enhanced cine steady-state free precession and T2-weighted CMR for assessment of ischemic myocardial areaat-risk in the presence of reperfusion injury. Int J Cardiovasc Imaging, 2019. 35(6): p. 1039-1045.

[19]. Kidambi, A., et al., Myocardial Extracellular Volume Estimation by CMR Predicts Functional Recovery Following Acute MI. JACC Cardiovasc Imaging, 2017. 10(9): p. 989-999.

[20]. Shao, X.N., et al., Texture analysis of magnetic resonance T1 mapping with dilated cardiomyopathy: A machine learning approach. Medicine (Baltimore), 2018. 97(37): p. e12246.

[21]. Gibbs, T., et al., Quantitative assessment of myocardial scar heterogeneity using cardiovascular magnetic resonance texture analysis to risk stratify patients post-myocardial infarction. Clin Radiol, 2018. 73(12): p. 1059.e17-1059.e26.

[22]. Bulluck, H., et al., Full left ventricular coverage is essential for the accurate quantification of the area-at-risk by T1 and T2 mapping. Sci Rep, 2017. 7(1): p. 4871.

\section{Tables}

Table 1 Study participant characteristics 


\begin{tabular}{|ll|}
\hline N=106 & Value \\
\hline Age & $61 \pm 11$ \\
\hline Male & $87(82 \%)$ \\
\hline BSA & $1.83 \pm 0.17$ \\
\hline Smoker & $30(28 \%)$ \\
\hline Family history & $11(10 \%)$ \\
\hline Hypertension & $63(59 \%)$ \\
\hline Hypercholesterolemia & $13(12 \%)$ \\
\hline Diabetes & $32(30)$ \\
\hline Killip (1-4) & 1 \\
\hline Pain to balloon time,h & $18.25 \mathrm{~h}$ \\
\hline Microvascular obstruction & $40(37.7 \%)$ \\
\hline Culprit vessel & \\
\hline LAD & 68 \\
\hline LCX & 10 \\
\hline RCA & 28 \\
\hline Affected vessels (num) & 1.93 \\
\hline TIMl pre-PCl $\geq 2$ & 33 \\
\hline Baseline CMR scan & $5 \mathrm{~d}$ \\
\hline Follow up CMR scan(n=45) & $102 \mathrm{~d}$ \\
\hline
\end{tabular}

Table 2 Distribution of the value of texture feature

\begin{tabular}{|c|c|c|c|c|c|c|c|c|}
\hline & \multicolumn{3}{|c|}{ all patients(106) } & \multicolumn{3}{|c|}{ patients with IMH or MVO(40) } & \multicolumn{2}{|c|}{ patients without IM } \\
\hline & infarction & salvage & remote & infarction & salvage & remote & infarction & s \\
\hline Mean & $71.19 \pm 15.93$ & $64.191 \pm 14.699 *$ & $56.517 \pm 10.379 * \#$ & $67.627 \pm 17.048$ & $66.105 \pm 12.215$ & $59.232 \pm 14.558$ & $73.310 \pm 14.958$ & $\epsilon$ \\
\hline Perc.50 & $67.68 \pm 13.97$ & $62.140 \pm 13.973^{*}$ & $53.308 \pm 7.727 \star \#$ & $64.625 \pm 15.840$ & $65.025 \pm 12.365$ & $54.975 \pm 9.857 * \#$ & $69.508 \pm 12.488$ & $\epsilon$ \\
\hline$(2,2)$ InvDfMom & $0.80 \pm 0.10$ & $0.782 \pm 0.228$ & $0.888 \pm 0.068^{\star} \#$ & $0.791 \pm 0.128$ & $0.812 \pm 0.153$ & $0.878 \pm 0.078^{\star} \#$ & $0.801 \pm 0.078$ & C \\
\hline (2,-2)AngScMom & $0.33 \pm 0.16$ & $0.433 \pm 0.235^{\star}$ & $0.573 \pm 0.218^{\star} \#$ & $0.312 \pm 0.149$ & $0.423 \pm 0.193$ & $0.549 \pm 0.224^{\star} \#$ & $0.339 \pm 0.171$ & C \\
\hline$(4,0)$ Entropy & $0.71 \pm 0.31$ & $0.447 \pm 0.271 *$ & $0.447 \pm 0.243^{\star}$ & $0.754 \pm 0.412$ & $0.501 \pm 0.290 *$ & $0.481 \pm 0.273^{\star}$ & $0.687 \pm 0.234$ & C \\
\hline 45dgrLngREmph & $15.13 \pm 9.90$ & $7.922 \pm 5.195^{\star}$ & $34.629 \pm 22.611^{\star} \#$ & $17.443 \pm 12.680$ & $7.958 \pm 5.168^{\star}$ & $34.723 \pm 29.800 * \#$ & $13.745 \pm 7.571$ & 7 \\
\hline 45dgr_Fraction & $0.37 \pm 0.11$ & $0.481 \pm 0.147 *$ & $0.261 \pm 0.077^{\star} \#$ & $0.355 \pm 0.119$ & $0.481 \pm 0.128^{\star}$ & $0.273 \pm 0.096 * \#$ & $0.375 \pm 0.105$ & C \\
\hline 135dr_GLevNonU & $61.96 \pm 26.03$ & $31.811 \pm 18.93^{*}$ & $99.839 \pm 26.231 * \#$ & $67.305 \pm 27.206$ & $35.399 \pm 18.899 *$ & $91.739 \pm 25.324 * \#$ & $58.770 \pm 24.956$ & 2 \\
\hline
\end{tabular}

${ }^{*} \mathrm{p}<0.001$, compared with infarction area

$\# p<0.001$, compared with salvage area

Table 3. AUC value between ROIs for features 


\begin{tabular}{|c|c|c|c|c|c|c|c|c|}
\hline & \multicolumn{3}{|c|}{ all patients(106) } & \multicolumn{3}{|c|}{ patients with IMH or MVO(40) } & \multicolumn{2}{|c|}{ patients without IMH or MVO(66) } \\
\hline & AUC邓inf/sal】 & AUC(sal/rem) & AUC(inf/rem) & AUC囚inf/sal囚 & AUC(sal/rem) & AUC(inf/rem) & AUC囚inf/sal囚 & AUC(sal/rem) \\
\hline Mean & $0.647 \pm 0.074$ & $0.735 \pm 0.068$ & $0.830 \pm 0.057$ & $0.516 \pm 0.128$ & $0.722 \pm 0.125$ & $0.696 \pm 0.119$ & $0.724 \pm 0.087$ & $0.741 \pm 0.085$ \\
\hline Perc.50 & $0.639 \pm 0.074$ & $0.771 \pm 0.065$ & $0.855 \pm 0.054$ & $0.522 \pm 0.129$ & $0.793 \pm 0.103$ & $0.741 \pm 0.111$ & $0.731 \pm 0.088$ & $0.764 \pm 0.082$ \\
\hline$(2,2)$ InvDfMom & $0.585 \pm 0.077$ & 0602 & 057 & $600 \pm 0.125$ & $0.682 \pm 0.118$ & $2 \pm 0$. & 0.099 & 090 \\
\hline$(2,-2)$ AngScMom & $0.647 \pm 0.074$ & $0.673 \pm 0.074$ & $0.807 \pm 0.060$ & $0.675 \pm 0.118$ & $0.662 \pm 0.122$ & $0.788 \pm 0.105$ & $0.630 \pm 0.094$ & $0.680 \pm 0.092$ \\
\hline$(4,0)$ Entropy & $0.759 \pm 0.074$ & $0.514 \pm 0.078$ & $0.776 \pm 0.063$ & $0.736 \pm 0.111$ & $0.541 \pm 0.128$ & $0.757 \pm 0.110$ & $0.774 \pm 0.077$ & $0.503 \pm 0.099$ \\
\hline 45dgrLngREmph & $0.791 \pm 0.061 *$ & $0.957 \pm 0.023^{*}$ & $0.841 \pm 0.053$ & $0.833 \pm 0.092^{*}$ & $0.934 \pm 0.052^{\star}$ & $0.757 \pm 0.107$ & $0.761 \pm 0.082$ & $0.971 \pm 0.021^{*}$ \\
\hline 45dgr_Fraction & $0.772 \pm 0.064^{*}$ & $0.934 \pm 0.034^{*}$ & $0.815 \pm 0.057$ & $0.827 \pm 0.093^{*}$ & $0.919 \pm 0.061^{*}$ & $0.742 \pm 0.109$ & $0.740 \pm 0.085$ & $0.942 \pm 0.040^{*}$ \\
\hline 135dr_GLevNonU & $0.845 \pm 0.052^{\star}$ & $0.980 \pm 0.015^{\star}$ & $0.861 \pm 0.050$ & $0.855 \pm 0.083^{*}$ & $0.942 \pm 0.041^{*}$ & $0.758 \pm 0.106$ & $0.845 \pm 0.066^{\star}$ & $0.988 \pm 0.020^{*}$ \\
\hline
\end{tabular}

${ }^{*} \mathrm{p}<0.001$, compared with Mean

Table 4. Prognosis prediction in follow-up CMR scans

\begin{tabular}{|c|c|c|c|c|c|c|}
\hline \multicolumn{3}{|c|}{ Logistics regression for LV remodeling } & \multicolumn{2}{|c|}{ Univariable linear regression for decreased EF } & \multicolumn{2}{|c|}{ Univariable linear regression for decreased strain } \\
\hline & OR value & $p$ value & $R$ value & $P$ value & $\mathrm{R}$ value & P value \\
\hline Sex & 0.897 & 0.931 & 0.135 & 0.376 & 0.031 & 0.839 \\
\hline Age & 0.980 & 0.534 & 0.347 & 0.019 & 0.073 & 0.633 \\
\hline BSA & 14.695 & 0.264 & 0.191 & 0.210 & 0.152 & 0.320 \\
\hline Diabetes & 0.784 & 0.752 & 0.070 & 0.646 & 0.050 & 0.742 \\
\hline Hypertension & 1.579 & 0.512 & 0.122 & 0.424 & 0.025 & 0.870 \\
\hline Hyperlipoidemia & 2.417 & 0.404 & 0.060 & 0.695 & 0.024 & 0.877 \\
\hline Smoker & 0.880 & 0.848 & 0.016 & 0.919 & 0.259 & 0.086 \\
\hline Family history & 0.867 & 0.875 & 0.105 & 0.492 & 0.065 & 0.672 \\
\hline PeakTNI & 0.981 & 0.086 & 0.158 & 0.299 & 0.053 & 0.729 \\
\hline TIMI & 0.852 & 0.537 & 0.047 & 0.076 & 0.179 & 0.240 \\
\hline Mean & 1.018 & 0.401 & 0.112 & 0.463 & 0.57 & 0.708 \\
\hline Perc.50 & 1.006 & 0.794 & 0.063 & 0.679 & 0.004 & 0.977 \\
\hline$S(2,2)$ InvDfMom & 2.692 & 0.737 & 0.120 & 0.431 & 0.053 & 0.727 \\
\hline$S(2,-2)$ AngScMom & 0.963 & 0.986 & 0.073 & 0.632 & 0.002 & 0.988 \\
\hline$S(4,0)$ Entropy & 1.032 & 0.968 & 0.043 & 0.779 & 0.030 & 0.847 \\
\hline 45dgr_LngREmph & 1.018 & 0.498 & 0.078 & 0.611 & 0.023 & 0.879 \\
\hline 45dgr_Fraction & 0.321 & 0.714 & 0.124 & 0.416 & 0.045 & 0.769 \\
\hline 135dr_GLevNonU & 0.986 & 0.279 & 0.151 & 0.321 & 0.077 & 0.614 \\
\hline
\end{tabular}

\section{Figures}




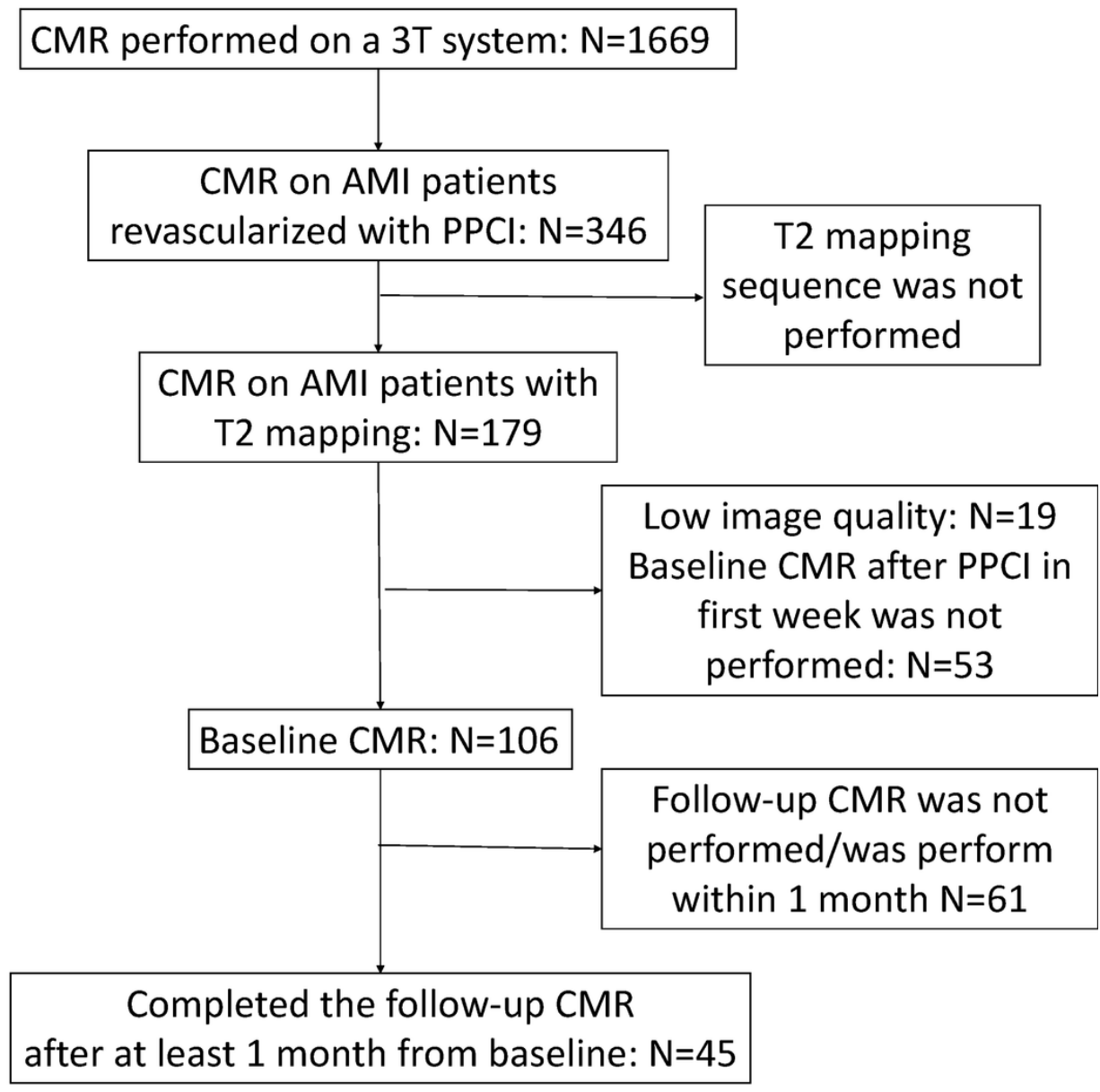

Figure 1

A study flowchart showing patient recruitment, inclusion and exclusion criteria for the study, reasons for patient dropout, and the number of final participants. $\mathrm{CMR}=$ cardiac $\mathrm{MR}, \mathrm{AMI}=$ acute myocardial infarction, $\mathrm{PPCl}=$ primary percutaneous coronary intervention 
a

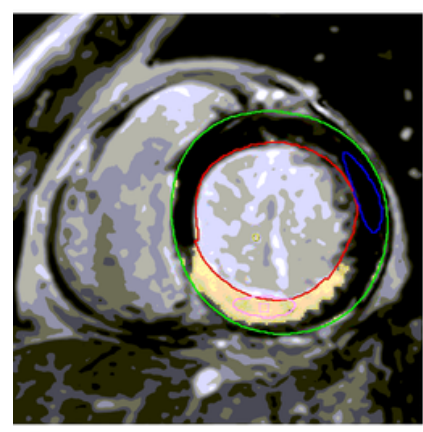

b

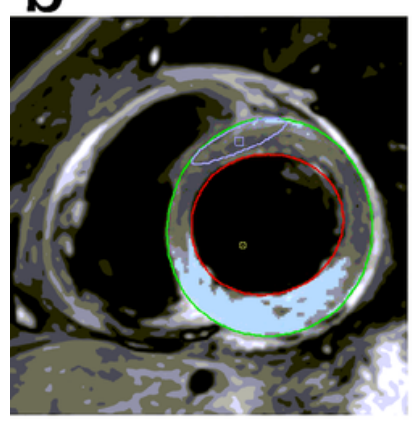

C

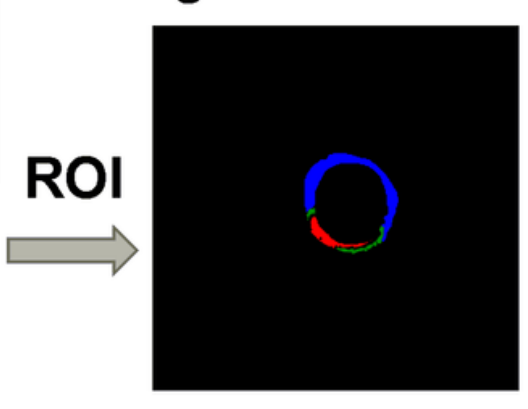

T2 mapping

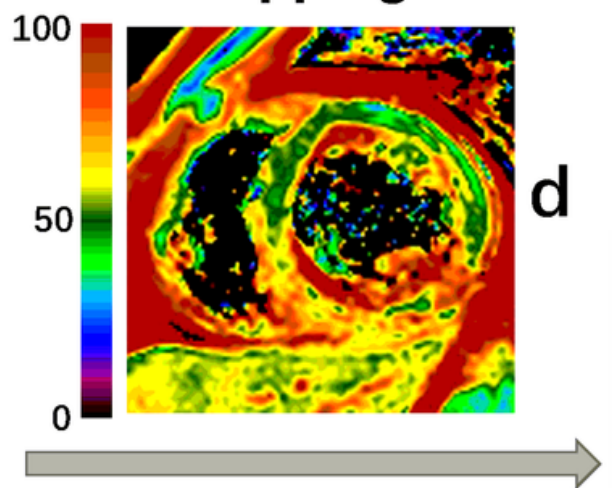

ROI\&T2 mapping

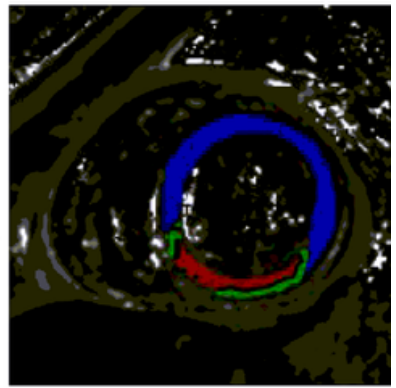

\section{Figure 2}

An illustration of the ROI drawing process. All four short-axis images were gathered from a 55-year-old man from the same position of the myocardium. In LGE imaging (a), the yellow part of the myocardium was identified as positive, while in T2-STIR (b) the blue part of the myocardium was identified as positive. Salvageable myocardium was identified as LGE negative and T2-STIR positive, and was then extracted and drawn by hand using T2 mapping, and the rest of the myocardial ring was defined as "remote" myocardium. The green circle represents epicardium; the red circle represents endocardium; the blue circle represents normal myocardium, the pink circle in (a) represents infarcted myocardium. In (c) and (d), blue represents remote myocardium, while red and green represent infarcted and salvageable myocardium. 


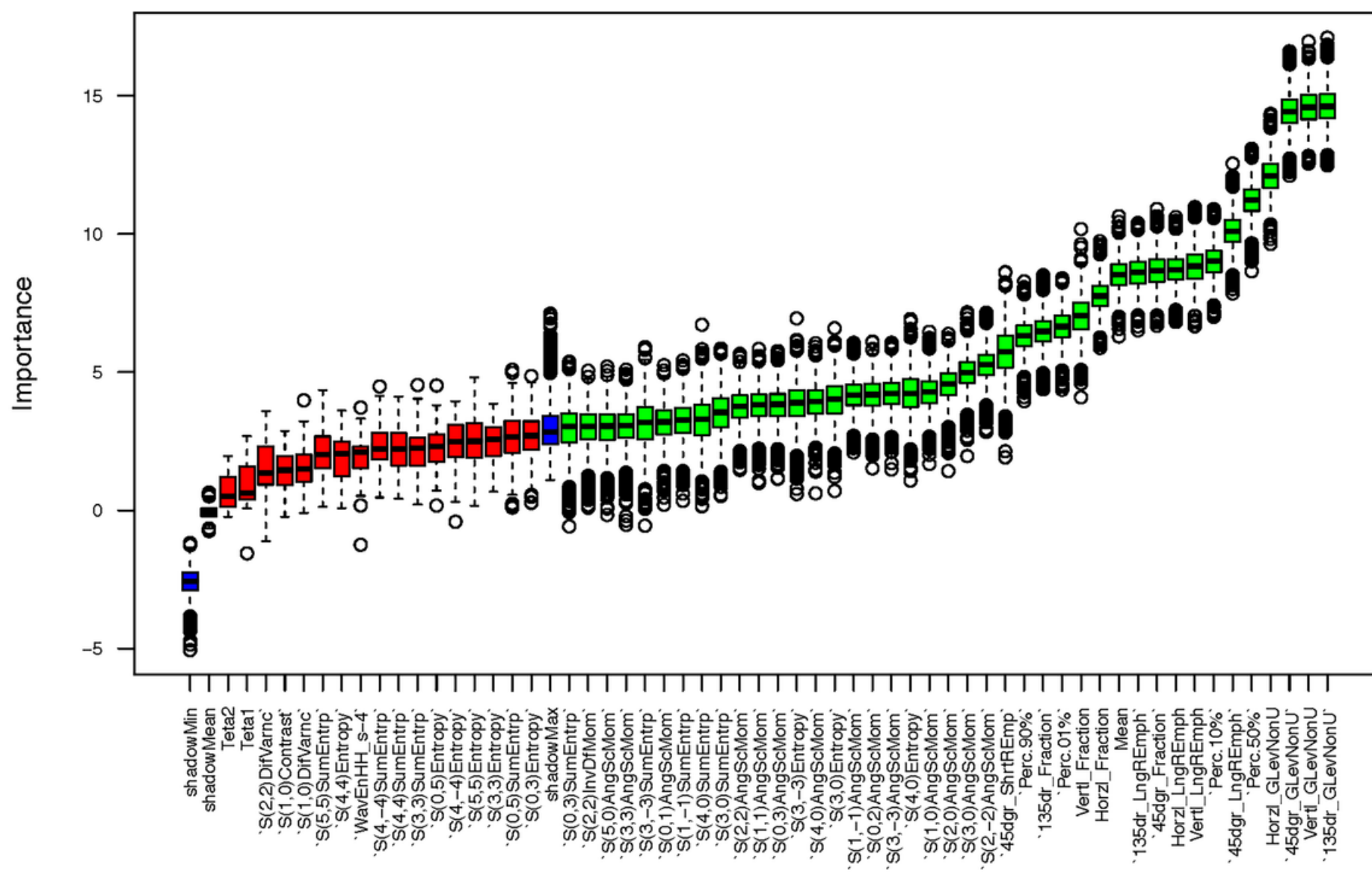

Figure 3

A correlogram illustrating auto- and cross-correlations of important texture features. Blue circles indicate a positive correlation, and red circles a negative correlation. The larger the circle and the darker the color, the higher the correlation between two features. The selected features were divided into seven subgroups according to this correlogram, and features were selected from each subgroup according to Fig.3. 


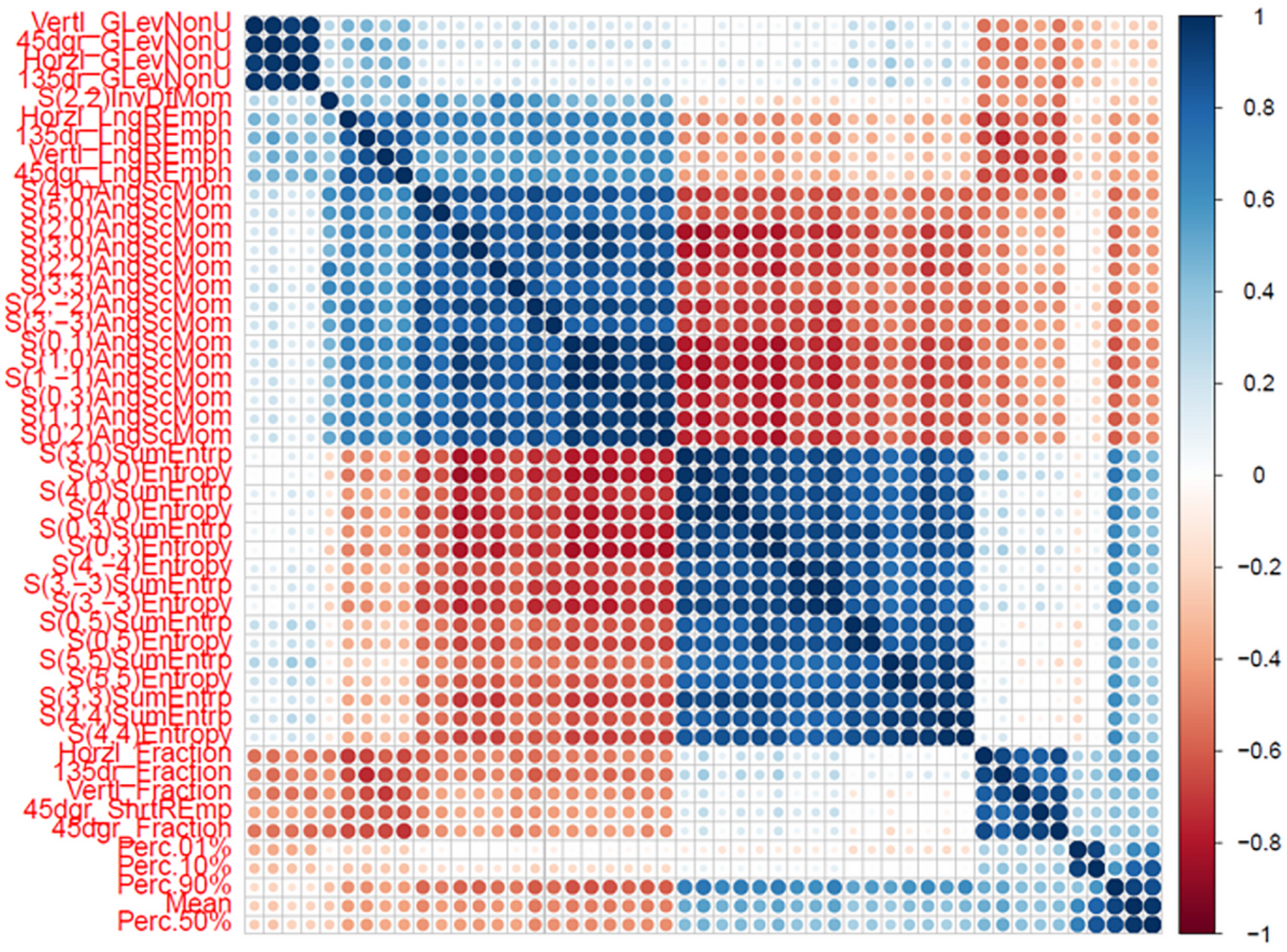

Figure 4

A correlogram illustrating auto- and cross-correlations of important texture features. Blue circles indicate a positive correlation, and red circles a negative correlation. The larger the circle and the darker the color, the higher the correlation between two features. The selected features were divided into seven subgroups according to this correlogram, and features were selected from each subgroup according to Fig.3. 

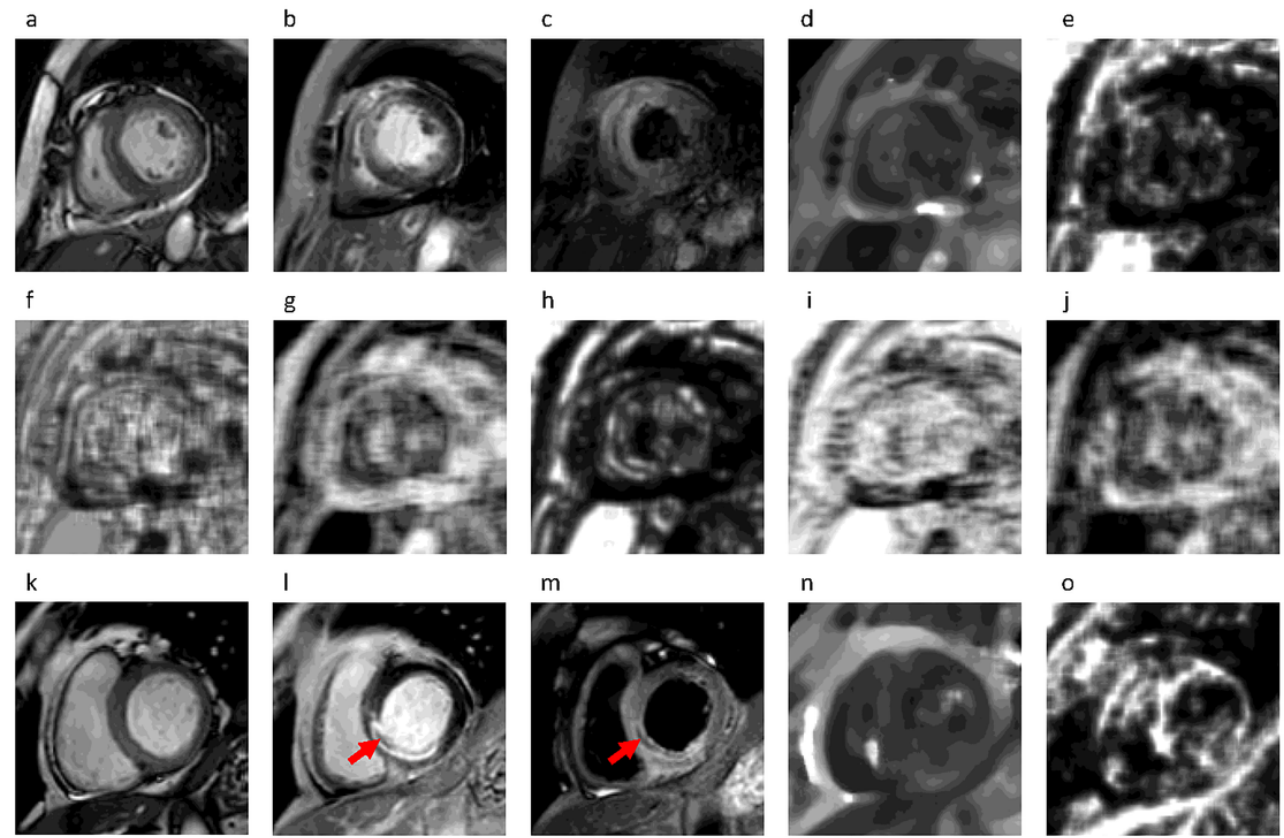

m
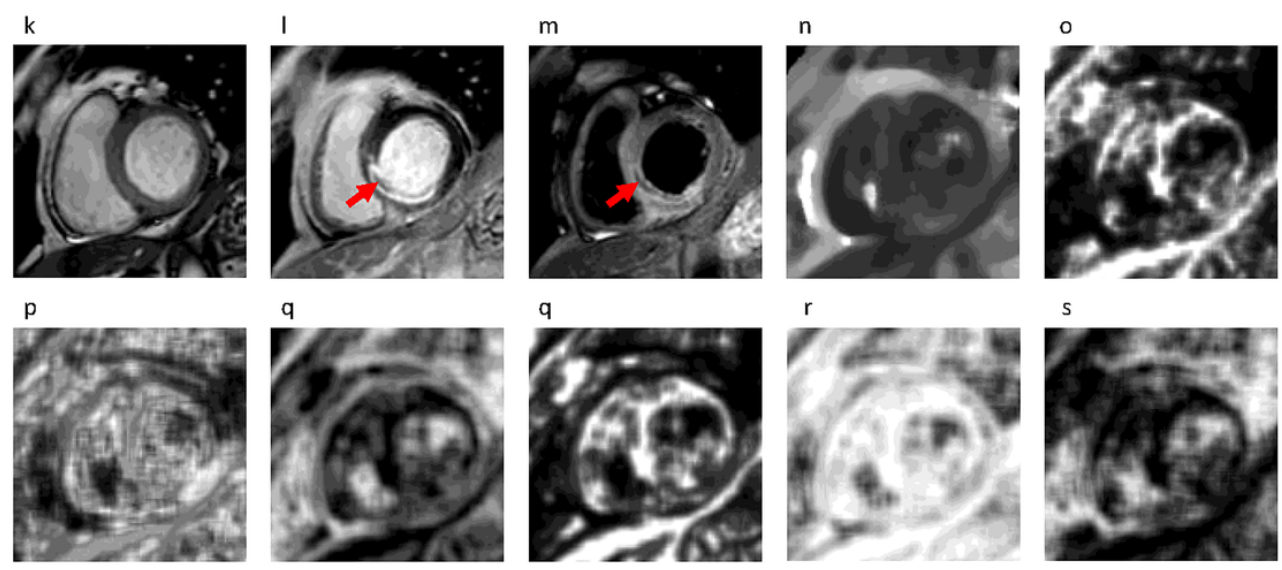

\section{Figure 5}

Examples of collected sequences and selected features of images from a patient without MVO (a 64 years-old female), and a patient with MVO and IMH (a 57 years-old male; MVO is indicated by the red arrowhead in T2-STIR(I) and LGE(m) imaging). MVO and IMH are represented as a black color lump inside the positive myocardium. In the order from (a) to (j) and (k) to (s) the images were from: cine, LGE, T2-STIR, Perc.50, 45dgrLngREmph, 135dr_GLevNonU,

$(4,0)$ Entropy, (2,-2)AngScMom, (2,2)InvDfMom and 45dgr_Fraction. 

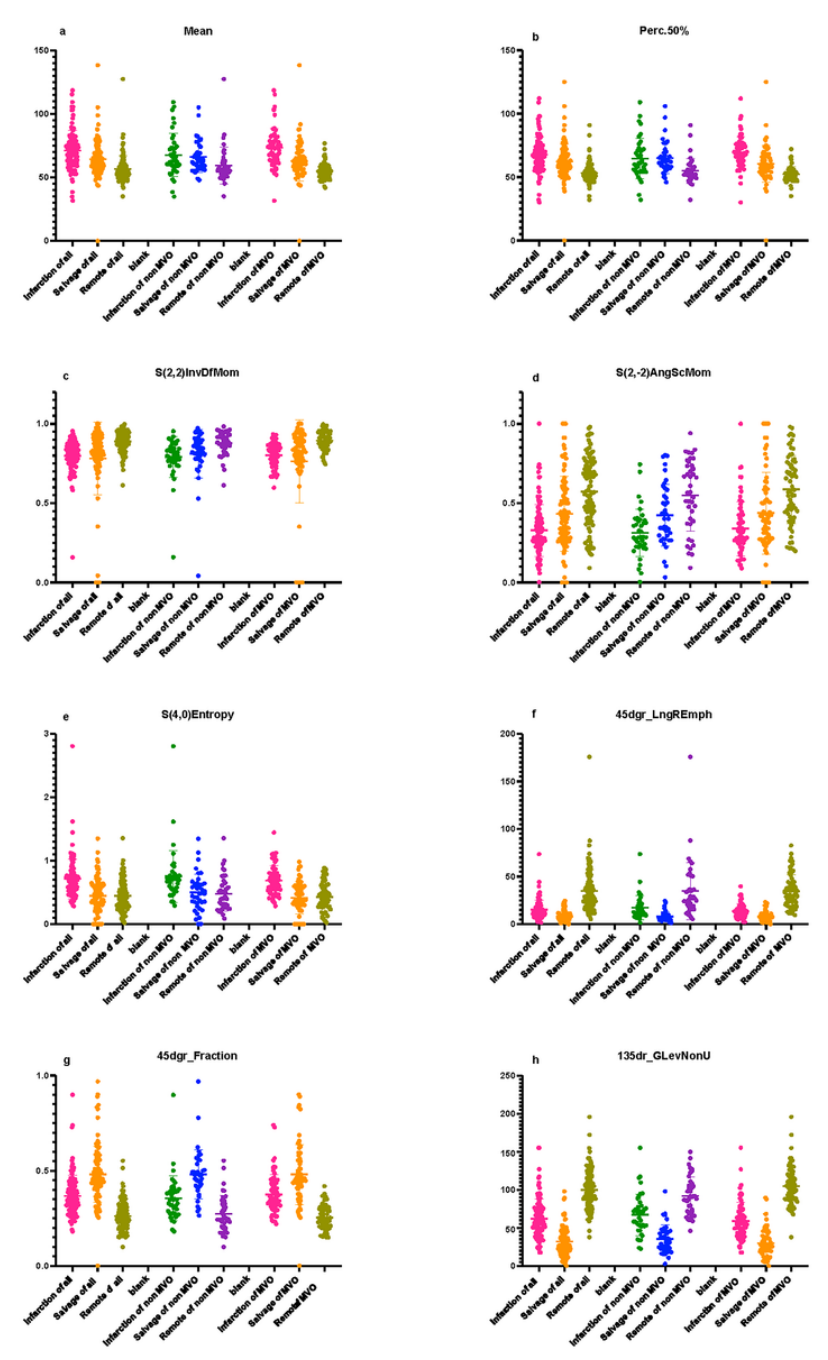

\section{Figure 6}

Scatter diagram for the selected texture features and mean of T2 mapping in infarcted, salvageable, and remote myocardium. In each category, features were plotted into three subgroups: i): all patients, ii) patients with MVO, iii) patients without MVO. In the order from (a) to (h): mean, Perc.50, (2,2)InvDfMom, (2,-2)AngScMom, (4,0)Entropy, 45dgrLngREmph, 45dgr_Fraction and 135dr_GLevNonU.

\section{Supplementary Files}

This is a list of supplementary files associated with this preprint. Click to download.

- OnlineAppendixFig.1.tif

- OnlineAppendixTable1.docx

- OnlineAppendixFig.1legends.docx 